\title{
Aspectos relacionados con el control del flujo secundario de agua en climatización centralizada
}

\author{
Aspects Related to the Control of the Secondary Flow of Water \\ in Centralized air Conditioning
}

\author{
Montero-Laurencio R. \\ Facultad de Metalurgia Electromecánica \\ Instituto Superior Minero Metalúrgico \\ Centro de Estudio de Energía y Tecnología Avanzada de Moa \\ Correo:rmontero@ismm.edu.cu
}

\author{
Hechavarría-Hernández J.R. \\ Centro de estudios CAD/CAM \\ Facultad de Ingeniería \\ Universidad de Holguin \\ Correo:jesus@cadcam.uho.edu.cu
}

Información del artículo: recibido: junio de 2011, aceptado: noviembre de 2011

\section{Resumen}

En este trabajo se realiza el ajuste del controlador proporcional integral incorporado en los variadores de velocidad utilizados en circuitos secundarios de agua fría en la climatización centralizada de un hotel. Quedó definida la ganancia proporcional y el tiempo de integración que garantiza un error mínimo de un $\mathrm{kPa}$ en el control de la presión. A partir de pruebas a lazo abierto se presenta un enfoque multivariable del sistema y se define la estructura de red neuronal artificial la cual predice el comportamiento de la presión, la potencia y temperatura de retorno, para un ajuste de $94 \%$. La diferencia del trabajo entre el lazo abierto y lazo cerrado del accionamiento, próximas al mismo punto de operación indican el ahorro de 13\% de energía eléctrica.

\section{Abstract}

In this paper the tuning of the proportional integral controller, incorporated in the variable frequency drive implemented on the secondary variable flow system of cold water of the centralized air conditioning system of a Hotel is carried out. The proportional gain and the time of integration that guarantee a minimum error of one $\mathrm{kPa}$ in the control of pressure were defined. A focus multi-variable of the system is presented. It is defined the structure of artificial neural network that better it predicts the behaviour of the pressure, the power and return temperature with an adjustment of $94 \%$. It is determined that there is a saving of $13 \%$ of electric power, in similar operation conditions, when using a control for closed loop with respect to a control for open loop.

\section{Descriptores}

- variadores de velocidad

- red neuronal artificial

- controlador proporcional integral

- circuitos secundarios de agua fría

- climatización centralizada

\section{Keywords}

- variable frequency drive

- artificial neural network

- Pl controller

- secondary variable flow

- centralized air conditioning 


\section{Introducción}

Se tienen estudios en donde se ha visto que el consumo de energía eléctrica en los hoteles se encuentra entre $85 \%$ y $95 \%$ (Montero y Góngora, 2008), de este consumo, $60 \%$ se utiliza en la climatización de las áreas de las instalaciones (Montelier, 2008; Armas, 2008). En Cuba, por existir un clima tropical, las instalaciones hoteleras tienen como una de sus principales necesidades el consumo de energía para la climatización de sus espacios, debiendo lograr determinados requisitos de confort, aprovechando al máximo los recursos energéticos.

En los sistemas de climatización centralizados por agua helada (SCCAH) para lograr que el agua fría llegue a cada unidad final se realiza el transporte del fluido mediante sistemas de bombeo denominados circuitos secundarios de agua fría (CSAF). Cerca de $80 \%$ de los hoteles de 4 y 5 estrellas cuentan con SCCAH en los cuales el líquido que se transporta recorre grandes distancias. Recientemente McQuiston et al. (2008) plantea que el agua por su alto calor específico, puede transportar mayor cantidad de energía por unidad de volumen que el aire, lo cual implica el uso de tuberías de menor diámetro.

Aproximadamente en $92 \%$ de los hoteles con climatización centralizada, tienen impulsión de agua fría a caudal constante, lo que implica un uso irracional de energía eléctrica (Montero, 2004). En los años 90 el bombeo consumía aproximadamente $18 \%$ de la energía de la climatización, mientras que en la primera década del siglo XXI, con las mejoras introducidas en las unidades enfriadoras, el consumo se incrementó a 26\% (Ryan, 2008). Estos datos demuestran la objetividad de lograr mejoras energéticas en estos sistemas.

Independientemente del tipo de caudal que experimentan los CSAF (constante o variable), existe un conjunto de parámetros que influyen en el comportamiento energético, entre ellos las características climatológicas de la región, la carga térmica de la edificación, la ocupación de los locales, las propiedades de las instalaciones, las características del accionamiento electromecánico y el equilibrado hidráulico. Todos estos aspectos han sido abordados de manera específica en diferentes literaturas dentro de las que se pueden mencionar a Petitjean (1997); Bonal (1999); Coad (2000); Lowery (2000) y Streeter (2000).

No todos los sistemas instalados presentan CSAF a flujo variable y para generalizar esta aplicación se hace necesario la elección de un variador de velocidad (VV), su preparación funcional y la evaluación de su desempeño ante diferentes condiciones de operación. Asociado al trabajo de los VV se encuentran los controles que lo go- biernan. Estos controles asimilan los parámetros para la regulación, pero en ocasiones no se cuenta con toda la información para calibrarlos. Por lo general, se emplean los parámetros que por defecto asigna el fabricante. Este elemento obliga a definir qué valores incorporar al controlador para mejorar la respuesta ante perturbaciones. Un modo de integrar las perturbaciones al control de los CSAF es presentar primero un enfoque multivariable y definir las relaciones que existen entre el conjunto de variables dependientes e independientes del sistema. Algunos trabajos como los Xinqiao (2007) y Zhenjun (2009) plantean esta estrategia, aportando soluciones específicas para los casos analizados.

\section{Desarrollo}

Los sistemas de impulsión de agua fría se diseñan para garantizar los caudales que demandan las condiciones máximas de carga térmica, pero la mayor parte del tiempo opera a una fracción de su potencia nominal. Por lo tanto, un sistema de control apropiadamente diseñado, debe mantener buenas condiciones ambientales interiores para cualquier condición predecible y con el menor costo posible durante el servicio de la instalación.

Aun cuando muchos controles electrónicos puedan funcionar en modo proporcional integral derivativo (PID), los buenos sistemas de control para sistemas de climatización generalmente no requieren la parte derivativa (McQuiston et al., 2008)

Los resultados que aparecen a continuación se basan en el desempeño del controlador instalado en un CSAF para la climatización de una edificación en un hotel, en el cual se utilizaron, de forma paralela, un sistema automático de medición (SAM) basado en el sistema EROS de supervisión y control (Núñez y Rodríguez, 2009) y el sistema TAC Vista de gestión integral de edificios (Schneider Electric, 2007). Estos sistemas sustituyeron la automatización anterior debido al deterioro que presentaba.

En la figura 1 se aprecia cómo a una ocupación constante en la edificación, aproximadamente de $90 \%$, se realizaron cambios en el valor deseado de la presión del sistema desde la computadora, con lo cual se logra que trabaje en todo su diapasón a lazo cerrado. El consumo de energía antes del montaje del SAM era excesivo, debido a que los sensores de presión estaban defectuosos, el sistema se operaba entonces a frecuencia constante de $35 \mathrm{~Hz}$. Una disminución sucesiva en el valor de consigna de la presión de envío exige una menor frecuencia para el trabajo del motor y por tanto menor demanda de potencia activa. Para comprobar el correcto funcionamiento del controlador se provocaron grandes per- 
turbaciones en el valor de consigna de la presión hasta estabilizarla en valores de 400, 420, 450 y $500 \mathrm{kPa}$.

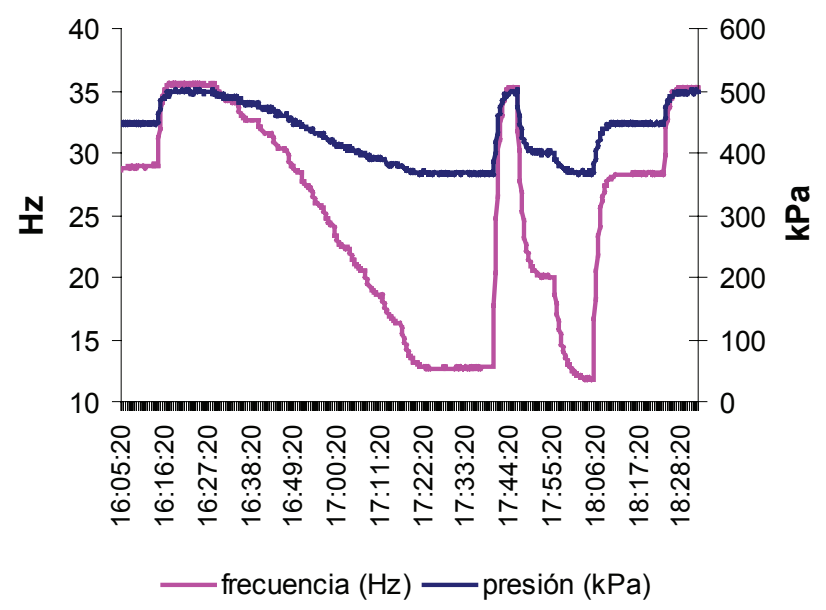

Figura 1. Respuesta de la frecuencia ante diferentes valores de consigna de presión de envío

La red hidráulica utilizada en los CSAF a caudal variable es mallada. En función de la ocupación de las habitaciones se demanda un caudal por cada unidad terminal ventiloconvectora (fancoil), lo que implica cambios en el comportamiento hidráulico de la instalación. Estos comportamientos aleatorios pueden apreciarse si el CSAF se opera a frecuencia constante en el VV. En la figura 2 se muestran los cambios en la dinámica del sistema a los cuales debe adaptarse el lazo de control cerrado proporcional integral (PI). Las mediciones se realizaron cada tres minutos, durante 24 horas, a una frecuencia constante de operación de $35 \mathrm{~Hz}$ y ocupaciones de $95 \%$ y $92 \%$.

La vigilancia de la presión de envío permite en los sistemas a caudal variable que el fluido sea proporcional a la cantidad de calor que debe extraerse. La diferencia de temperatura entre el envío y el retorno, a pesar de ser prácticamente constante, refleja el grado de intercambio térmico con el edificio. La carga térmica del edificio está influenciada por las condiciones me- teorológicas y la ocupación de locales por lo que es variable durante el día.

La función del control se activa mediante la asignación de una entrada analógica en el retorno PI del VV. Esta variable se obtiene por medición directa a través de un sensor de presión a la salida de la bomba. En el caso de la elección del tipo de ley tensión/frecuencia se escogió la ley de par variable, la cual se aplica a bombas y ventiladores (Schneider Electric, 2008). Tomando en consideración las recomendaciones del fabricante para la sintonía del regulador PI, se realizaron pruebas en el CSAF que permitieron obtener resultados de la sintonía del controlador para las diferentes bombas en funcionamiento (bomba de zona 3, bomba de zona 4 y bomba de zona 5). Se tomaron los valores de ocupación de cada zona para verificar su repercusión en la red hidráulica y se eligieron los valores de la ganancia proporcional y los tiempos de integración para cada bomba. El valor de presión elegido inicialmente fue de $500 \mathrm{kPa}$, según establecen las condiciones de diseño en el expediente de obra del hotel. Posteriormente se apagó el sistema hasta llegar a un punto de reposo y luego se realizaron arranques con lo cual se pudo determinar la respuesta del controlador ante esta perturbación (la más severa).

De los resultados, se seleccionaron las mejores ganancias. También se cambió el valor de la consigna de la presión en las bombas para ver las respuestas ante otros valores de referencia. En la figura 3 se muestran los resultados de sintonía para la bomba de la zona 5 . El mejor tiempo de establecimiento de la presión se logró en periodos que oscilan entre 26 y 31 segundos. Estos resultados permitieron encontrar el mejor desempeño del controlador con valores de ganancia proporcional de uno y ganancia integral de 1.3 segundos generalizables para todas las bombas.

Una vez establecidos los parámetros de los controladores, se realizaron pruebas de funcionamiento bajo distintas condiciones de ocupación de las zonas y diferentes valores de consigna. En la figura 4 se aprecian los resultados de las pruebas realizadas en la zona 5 para

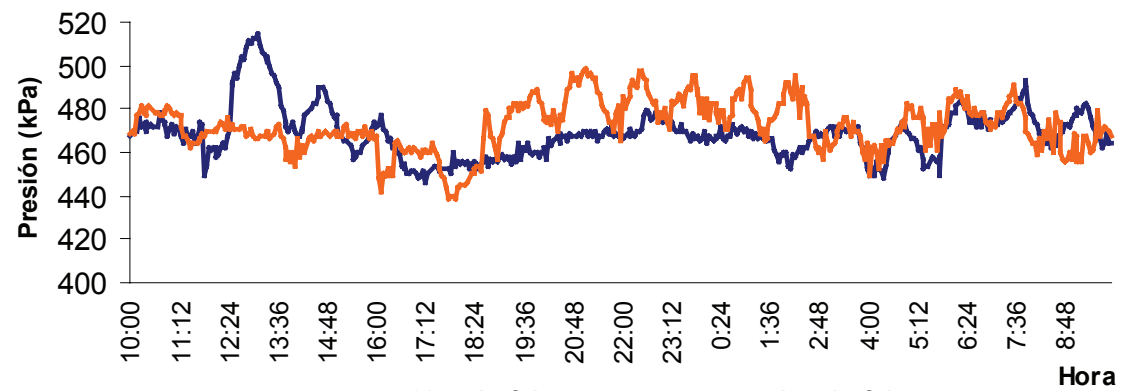

Figura 2. Gráficos de presión a caudal constante para dos días de trabajo de un CSAF 
días específicos. Esta zona es la más cercana a las estaciones de bombeo de la sala de máquinas. La ocupación de esta zona durante los días de pruebas fue 97\% (500 $\mathrm{kPa}), 91 \%$ (470 kPa) y 50\% (440 kPa). El motor acoplado a la bomba es de $4.5 \mathrm{~kW}$.

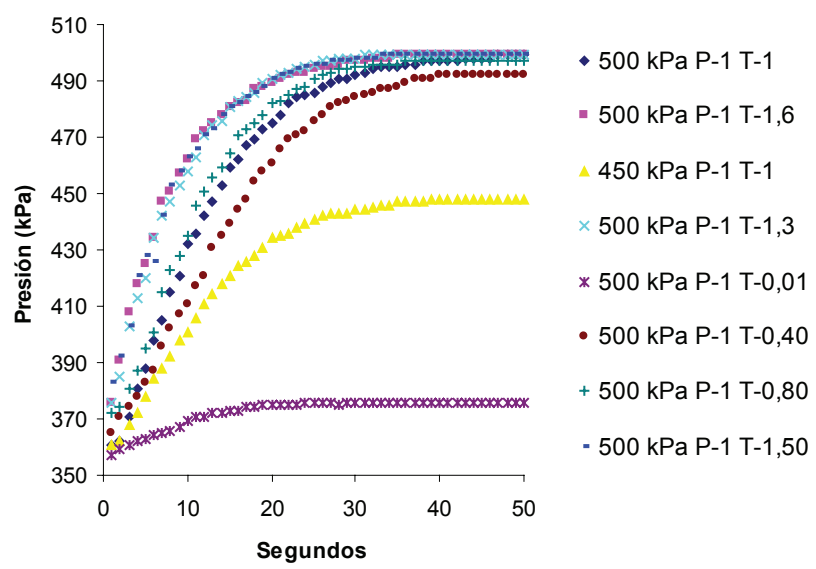

Figura 3. Repuestas para diferentes valores de consigna y parámetros del controlador PI en una bomba

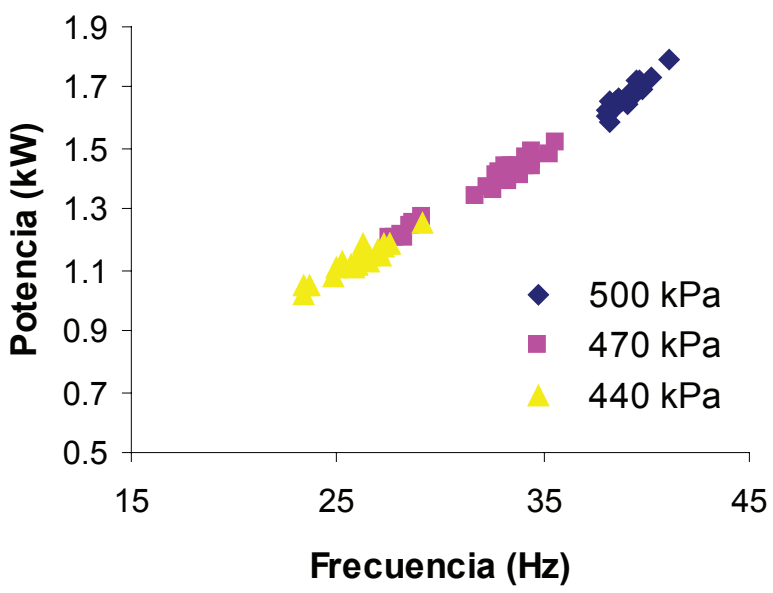

Figura 4. Relación entre frecuencia y potencia a diferentes valores de consigna

Debido a la variación de la carga térmica total existe una variación en el torque del motor de la bomba independientemente del valor de consigna de la presión. Estos cambios en la carga térmica significan conexión y desconexión de fancoil. Este cambio de ocupación de los locales provoca cambios en la resistencia hidráulica de la red mallada y como consecuencia varia la cantidad de flujo de agua.

Para demostrar la diferencia energética entre el régimen a flujo constante y a flujo variable, se prefijó la frecuencia a $32.5 \mathrm{~Hz}$ con presión de envío de $450 \mathrm{kPa}$ en una zona de habitaciones del hotel. Luego se desconectaron las baterías verticales de fancoil en intervalos de 5 minutos, logrando cantidades de fancoil de 59, 53, 47, 37,31 y 25 en forma ascendente y descendente.

En la figura 5 pueden verse los resultados de la demanda de energía eléctrica para cambios de ocupación en regímenes de flujo constante (lazo de control abierto) y de flujo variable (lazo de control cerrado). Se aprecian las ecuaciones de correlación para ambos casos. La diferencia entre los valores medios de potencia fue $13 \%$.

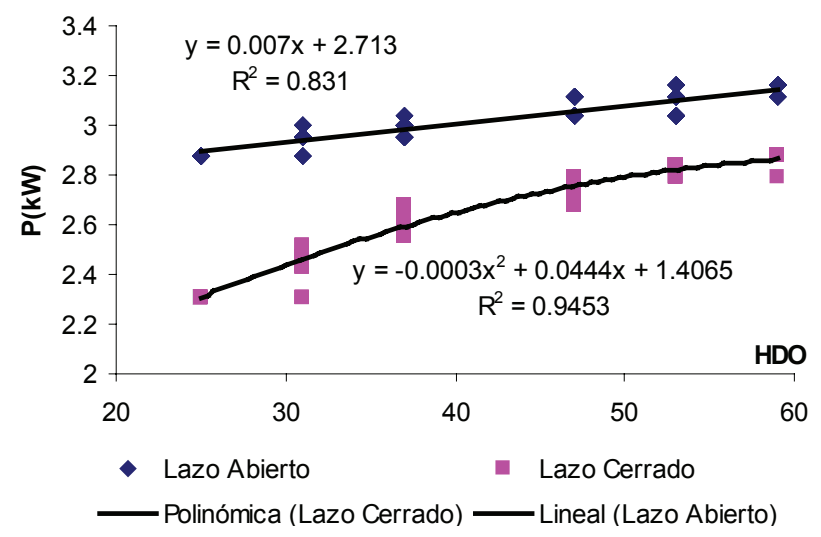

Figura 5. Diferencia entre la demanda de potencia a lazo abierto y a lazo cerrado

Para asegurar que el fluido llegue con todos sus requerimientos a los lugares más críticos no basta proponer un punto de operación del sistema teniendo en cuenta sólo la ocupación. El comportamiento térmico del edificio, influenciado por la temperatura ambiente exterior (Tamb) y la temperatura de envío del agua fría (Te), debiera tomarse en cuenta en la selección de un nuevo valor de consigna.

Cuando las condiciones exteriores se caracterizan por bajas temperaturas no es necesaria la misma cantidad de agua para extraer el calor de los locales por lo que una presión más baja resulta racional. También cuando la temperatura del agua de envío es menor, se logra el confort en menor tiempo. Estos aspectos conducen a integrar, en un análisis mutivariable, el comportamiento de los CSAF, lo cual permitiría mejorar los controles existentes de este tipo de accionamiento.

Para obtener una aproximación a la modelación multivariable se escogen las variables de entrada: habitaciones días ocupadas (HDO), Te y Tamb.

Las variables Te y Tamb se consideran además perturbaciones, por ser variables de coordinación (Arzola, 2000 y Hechavarría, 2009) del sistema de mayor envergadura impuesto por las enfriadoras y el comportamiento del clima. Las variables de salida son de- 
manda de potencia eléctrica del motor de la bomba $(\mathrm{P})$, la presión de envío $(\mathrm{Pe})$ y la temperatura de retorno del agua (Ts). En la figura 6 se muestra el gráfico del flujo de señales.

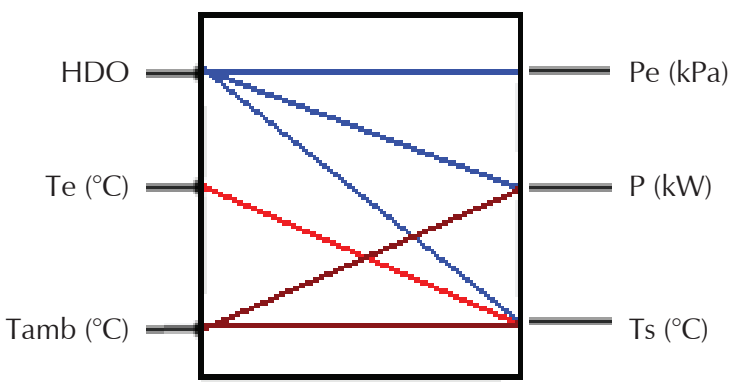

Figura 6. Enfoque multivariable de un CSAF

Antes de las pruebas para la modelación se seleccionó una de las zonas, se liberó el aire contenido en las redes para mejorar el transporte y se compensó el circuito hidráulico completando el volumen de agua necesario. Luego se trabajó el sistema a lazo abierto donde se limitó el valor máximo de frecuencia (registro HSP) para poder operar manualmente el VV y se prefijó una frecuencia máxima de $32.5 \mathrm{~Hz}$, equivalente a una presión en el sistema de $450 \mathrm{kPa}$ para las condiciones máximas (todas las unidades terminales conectadas).
Primero se obtuvo un modelo paramétrico con ayuda de la herramienta de Identificación del Matlab 7.7.0. Para la estructura del sistema propuesto esta herramienta sólo pudo estimar los modelos paramétricos ARX de segundo orden y el modelo en variable de estado. Los mejores ajustes para cada una de las salidas del modelo ARX al manipular las HDO fueron las siguientes: $40.43 \%$ para la presión de descarga de la bomba; $66.16 \%$ para la potencia eléctrica y $23.95 \%$ en la temperatura de retorno. Se obtuvieron ajustes muy similares para el modelo en variables de estado.

Como los ajustes no son significativos se realizó una modelación con ayuda de las redes neuronales artificiales (RNA). La función de aprendizaje escogida fue trainlm (Levenberg-Marquardt). La estructura de RNA que mejores resultados ofreció fue la Feedfoward Backpropagation. Se emplearon tres capas de neuronas. La primera capa cuenta con tres neuronas, con una función de transferencia tansig.

La capa intermedia es de 9 neuronas con la misma función tansig y la última capa es de 3 neuronas con la función de transferencia pureline. El aprendizaje se logró a las 51 iteraciones, con un error mínimo de validación de $9.2 \times 10^{-5}$. Como se observa en la figura 7 , el coeficiente de correlación entre los datos de salida medidos y los estimados por la RNA es 0.94 para los datos de entrenamiento, prueba y validación.
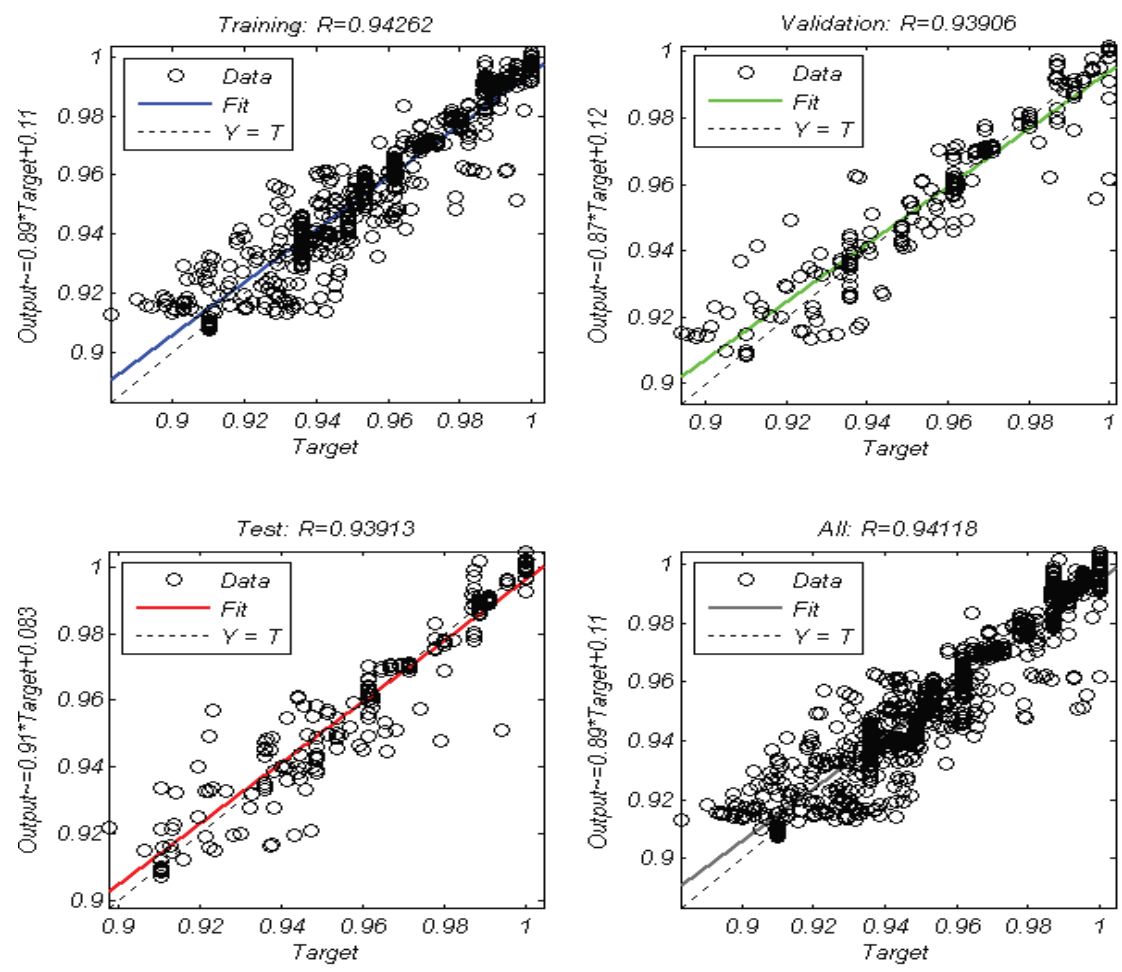

Figura 7. Resultados del proceso entrenamiento, pruebas y validación de la RNA 


\section{Conclusiones}

Para las condiciones críticas durante el arranque se obtuvieron los parámetros fundamentales del controlador PI incorporados en los VV de las diferentes secciones de bombeo con valores de ganancia proporcional $1 \mathrm{y}$ tiempo de integración 1.3 segundos con tiempos de establecimiento de la presión entre 25 y 31 segundos y un error máximo de $1 \mathrm{kPa}$.

El personal de mantenimiento del hotel cuenta con un material técnico para enfrentar las principales incidencias y manipulaciones que se deben realizar con los variadores de velocidad en los CSAF.

La diferencia entre los valores medios de potencia entre el trabajo a caudal constante y caudal variable alrededor del mismo punto de operación y para una misma situación de la ocupación en el caso de estudio es 13\%.

Se logró identificar un modelo multivariable basado en RNA que caracteriza el comportamiento de las variables presión de envío, potencia y temperatura de retorno en los CSAF a partir de las entradas HDO, temperatura de entrada del agua y temperatura ambiente.

\section{Agradecimientos}

A los ingenieros Maikel Núñez, Yosvani Rodríguez y Yosvany García por toda su colaboración durante su etapa de estudiante. Al ingeniero Víctor Peña por toda su experiencia. A la ingeniera Carmen por permitirme emplear el software EROS generado por su empresa. A los trabajadores de Servicios Técnicos del hotel caso de estudio.

\section{Referencias}

Armas-Valdez J.C. Procedimiento para la optimización de sistemas de climatización centralizados por agua helada desde la etapa de diseño conceptual, tesis (doctorado en ciencias técnicas), Cuba, Universidad de Cienfuegos, 2008, $100 \mathrm{p}$.

Arzola-Ruiz J. Sistemas de Ingeniería, ed. Félix Varela, La Habana, Cuba, 2000, pp. 5-30.

Bonal J. Accionamientos eléctricos a velocidad variable, volumen 1, fundamentos de electrotécnia y de mecánica, las técnicas de variación de velocidad, TEC \& DOC Editions, 1999, 413 p.

Coad W.J. Hidronic Heating and Cooling System Design, ASHRAE Handbook 2000, capítulo 12, 18 p.

Hechavarría J.R. Optimización del diseño de redes de distribución de agua bajo criterios técnico-económicos, tesis (doctorado en ciencias técnicas), Cuba, Universidad de Holguin, 2009, 130 p.

Lowery T., Peterson S.L., Swan E.J. Motors, Motor Controls and Variable-Speed Drives, ASHRAE Handbook 2000, capítulo 40, $14 \mathrm{p}$.
McQuiston F., Parker J., Spitler J. Calefacción ventilación y aire acondicionado: análisis y diseño, 1a ed., México DF, LIMUSA, 2008, pp. 22-27.

Montelier-Hernández S. Reducción del consumo de energía en instalaciones con sistemas de climatización centralizadas todo agua a flujo, tesis (doctorado en ciencias técnicas), Cuba, Universidad de Cienfuegos, 2008, 105 p.

Montero-Laurencio R., Góngora-Leyva E. Aplicación del Toolbox de identificación de Matlab en la estimación de gestión total eficiente de energía en Moa, Holguín, Cuba. Ingeniería, Investigación y Desarrollo, volumen 7 (número 2), 2008: 15-19.

Montero-Laurencio R. Disminución del consumo energético en los circuitos secundarios de agua fría de la climatización centralizada de hoteles, tesis (maestría en electromecánica), Cuba, Instituto Superior Minero Metalúrgico de Moa, 2004, 98 p.

Núñez M., Rodríguez Y. Sistema automático de medición para variables termohidráulicas en la climatización centralizada, tesis (ingeniería eléctrica), Cuba, Instituto Superior Minero Metalúrgico de Moa, 2009, 92 p.

Petitjean R. Total Hydronic Balancing, TA HYDRONICS, 1997, $530 \mathrm{p}$.

Ryan W. A Look at Chilled Water System Design, en: Conferencia de la firma Trane, 2008,

Schneider Electric. TAC Building Automation, TAC Vista 5, 2007.

Schneider Electric. Variadores de velocidad para motores asíncronos Altivar 31: Guía de programación, 2008, 75 p.

Streeter V., Benjamin E., Bedford K. Mecánica de fluidos, 9a ed., Santafé de Bogotá, McGraw-Hill, Best Seller International SA, 2000 [en línea]. Disponible en: http://www.mhhe.com

Xinqiao J. Energy Evaluation of Optimal Control Strategies for Central VWV Chiller Systems. Applied Thermal Engineering, volumen 27 (números 5-6), abril 2007: 934-941.

Zhenjun Ma., Shengwei W. Energy Efficient Control of Variable Speed Pumps in Complex Building Central Air-Conditioning Systems. Energy and Buildings (número 41), 2009: 9.

\section{Este artículo se cita:}

\section{Citación Chicago}

Montero-Laurencio, Reineris, Jesús Rafael Hechavarría-Hernández. Aspectos relacionados con el control del flujo secundario de agua en climatizacion centralizada. Ingeniería Investigación y Tecnología, XIII, 03 (2012): 307-313.

\section{Citación ISO 690}

Montero-Laurencio R., Hechavarría-Hernández J.R. Aspectos relacionados con el control del flujo secundario de agua en climatizacion centralizada. Ingeniería Investigación y Tecnología, volumen XIII (número 3), julio-septiembre 2012: 307-313. 


\section{Semblanzas de los autores}

Reineris Montero-Laurencio. Profesor asistente del Centro de Estudio de Energía y Tecnología Avanzada de Moa, ingeniero eléctrico, maestro en ingeniería electromecánica. Cursa el doctorado curricular de electromecánica. Coordinador de la maestría en eficiencia energética. Consultor en temas de gestión y eficiencia energética. Experiencia de ocho años como coordinador de proyectos en Oficina de Transferencias de Tecnología. Miembro de la Red Nacional de Eficiencia Energética del Ministerio de Educación Superior, de la Asociación Nacional de Economistas y de la Asociación Nacional de Arquitectos e Ingenieros de la Construcción. Posee dos premios anuales provinciales a la innovación tecnológica.

Jesús Rafael Hechavarría-Hernández. Profesor titular del Centro de Estudios CAD/CAM, ingeniero mecánico, maestro en CAD/CAM. Doctor en ciencias técnicas. Profesor invitado en varias universidades latinoamericanas. Realizó servicios científico-técnicos en empresas nacionales y extranjeras. Ha recibido más de 25 premios por su actividad científica. Es miembro de diferentes claustros de maestrías. Ha participado en varias redes y proyectos de alcance internacional. Es experto y consultor para el desarrollo de la industria mecánica, Miembro de la Unión Nacional de Arquitectos e Ingenieros de la Construcción; de la Asociación Nacional de Matemática y de la Asociación Nacional de Reconocimiento de Patrones en Cuba. 